\title{
Viabilidade econômica de diferentes sistemas de sangria em clones de seringueira
}

\author{
Juliano Quarteroli Silva ${ }^{(1)}$, Mario Ivo Tavares de Souza(2), Paulo de Souza Gonçalves ${ }^{(1)}$, \\ Adriano Tosoni da Eira Aguiar(1), Lígia Regina Lima Gouvêa ${ }^{(1)}$ e Raquel Nakazato Pinotti( ${ }^{(3)}$
}

\begin{abstract}
(1)Instituto Agronômico, Programa Seringueira, Caixa Postal 28, CEP 13001-970 Campinas, SP. E-mail: julianoufrrj@yahoo.com.br, paulog@iac.sp.gov.br, aguiar@iac.sp.gov.br, Igouvea@iac.sp.gov.br(2)Fazenda Santa Gilda, Grupo Rodobéns Agrícola e Pecuária Ltda., Rod. Marechal Rondon, Km 565, CEP 16700-000 Guararapes, SP. E-mail: marioivo@uol.com.br (3) Apta Regional Centro Oeste, Av. Rodrigues Alves, no 40, Horto Florestal, CEP 17030-000 Bauru, SP. E-mail: raquelnakazato@aptaregional.sp.gov.br
\end{abstract}

Resumo - O objetivo deste trabalho foi avaliar o desempenho produtivo e os aspectos econômicos de três clones de seringueira [Hevea brasiliensis (Willd. ex Adr. de Juss.) Muell. Arg.], sob nove sistemas de sangria. $\mathrm{O}$ experimento foi instalado sob delineamento de blocos ao acaso com parcelas subdivididas no tempo. Os tratamentos principais foram os clones PR 255, RRIM 600 e GT 1, submetidos aos seguintes sistemas de sangria: $1 / 2 \mathrm{~S} \mathrm{~d} / 36 \mathrm{~d} / 7.11 \mathrm{~m} / \mathrm{y} . \mathrm{ET} 2,5 \%$ Pa La 8/y; $1 / 2 \mathrm{~S} \mathrm{~d} / 3$ 6d/7.11m/y.ET 5,0\% Pa La 8/y; $12 \mathrm{~S} \mathrm{~S} / 46 \mathrm{~d} / 7.11 \mathrm{~m} / \mathrm{y} . \mathrm{ET} 2,5 \%$ Pa La 8/y; $1 / 2 \mathrm{~S} \mathrm{~d} / 46 \mathrm{~d} / 7.11 \mathrm{~m} / \mathrm{y}$.ET 5,0\% Pa La 8/y; 1/2S d/5 6d/7.11m/y.ET 2,5\% Pa La 8/y; $12 \mathrm{~S}$ d/5 6d/7.11m/y.ET 5,0\% Pa La 8/y; $1 / 2 \mathrm{~S} \mathrm{~d} / 7$ 6d/7.11m/y.ET 2,5\% Pa La 8/y;1/2S d/7 6d/7.11m/y.ET 5,0\% Pa La 8/y e 1/2S d/2 6d/7.11m/y (testemunha). As variáveis estudadas foram: perímetro do caule, produtividade de borracha seca e secamento do painel. Também foi avaliada a viabilidade econômica dos sistemas de sangria. Observaram-se maior produtividade e rentabilidade dos sistemas $1 / 2 \mathrm{~S}$ d/3.ET 2,5\% 8/y para os clones PR 255 e RRIM 600 e $1 / 2 \mathrm{~S}$ d/7.ET 2,5\% 8/y para o clone GT 1, comparados com a testemunha. A maior e a menor porcentagem de secamento do painel foram observadas nos sistemas $1 / 2 \mathrm{~S}$ d/3 ET 5,0\% 8/y e $1 / 2 \mathrm{~S}$ d/7.ET 5,0\% 8/y, respectivamente.

Termos para indexação: Hevea brasiliensis, economia, borracha natural, estimulação, secamento do painel.

\section{Economic viability of different tapping systems in rubber tree clones}

\begin{abstract}
The objective of this work was to evaluate yield performance and economic aspects of three clones of rubber tree [Hevea brasiliensis (Willd. ex Adr. de Juss.) Muell. Arg.], in different tapping systems. The trial was placed under randomized blocks design with split-plot in time. The main treatments were PR 255, RRIM 600 and GT 1 clones submitted to nine systems of tapping: $1 / 2 \mathrm{~S} \mathrm{~d} / 3$ 6d/7.11m/y.ET 2,5\% Pa La 8/y; 1/2S d/3 6d/7.11m/y.ET 5,0\% Pa La 8/y; 1/2S d/4 6d/7.11m/y.ET 2,5\% Pa La 8/y;1/2S d/4 6d/7.11m/y.ET 5,0\% Pa La 8/y;1/2S d/5 6d/7.11m/y.ET 2,5\% Pa La 8/y; $1 / 2 \mathrm{~S} \mathrm{~d} / 5$ 6d/7.11m/y.ET 5,0\% Pa La 8/y; $1 / 2 \mathrm{~S}$ d/7 6d/7.11m/y.ET 2,5\% Pa La 8/y;1/2S d/7 6d/7.11m/y.ET 5,0\% Pa La 8/y and $1 / 2 \mathrm{~S} \mathrm{~d} / 26 \mathrm{~d} / 7.11 \mathrm{~m} / \mathrm{y}$ (check). The variables were: girth increment, dry rubber productivity and brown bast. The economic viability of the tapping systems was also evaluated. Economical benefits in the $1 / 2 \mathrm{~S} d / 3$.ET 2,5\% 8/y system for PR 255 and RRIM 600 clones and in the $1 / 2 \mathrm{~S}$ d/7.ET 2,5\% 8/y system for GT 1 clone were observed, compared with the check. Highest and smaller incidence of brown bast were observed in the $1 / 2 \mathrm{~S} d / 3$.ET 5,0\% 8/y and $1 / 2 \mathrm{~S} d / 7$.ET $5,0 \% 8 / \mathrm{y}$ systems, respectively.
\end{abstract}

Index terms: Hevea brasiliensis, economy, natural rubber, stimulation, brown bast.

\section{Introdução}

A explotação do seringal tem como finalidade a obtenção do látex, sua retirada do seringal e sua conservação, de forma a colocá-lo em condições de ser beneficiado. Segundo Bernardes et al. (2000), a introdução dos clones de seringueira exige uma definição mais específica sobre o sistema de explotação a ser adotado, pois existe variação acentuada no comportamento de cada clone quando submetido a diferentes sistemas de sangria. Esses autores relatam que existem diferentes respostas de produção e longevidade do seringal que se relacionam com fatores como a idade para iniciar a sangria, esquema de estimulação adotado, utilização ou não de balanceamento do painel de sangria, tamanho e tipo de corte adotado.

A sangria da seringueira é uma das práticas mais importantes da cultura, pois além de ser um fator que determina a vida útil do seringal e a produtividade, responde 
por aproximadamente $60 \%$ dos custos totais da borracha produzida (Gonçalves et al., 2000).

De acordo com Gonçalves et al. (2000), um dos principais problemas encontrados na heveicultura do Estado de São Paulo é o alto custo da extração de borracha, que envolve mão-de-obra especializada. Em locais onde a disponibilidade de mão-de-obra qualificada é escassa, há tendência de adoção de sistemas de explotação com frequiência de sangria reduzida, tendo em vista a otimização dos serviços desses trabalhadores, obtendo-se, assim, aumento da produtividade e, conseqüentemente, maior rendimento financeiro líquido.

Outro problema nos sistemas de exploração comercial da seringueira é a ocorrência de seca do painel ("brown bast") em árvores em sangria. Este é um distúrbio fisiológico encontrado nas plantações, e ocorre na fase de sangria com o bloqueio do fluxo de látex contido no interior da casca do caule; algumas árvores cessam a produção em certas partes ou mesmo na totalidade da casca. Esse distúrbio pode ser causado pela adoção de sistemas de explotação com alta frequiência de sangria e altas concentrações de estimulante (Gonçalves et al., 2001). Se o número de plantas com secamento ou a média de porcentagem seca dos painéis aumentar, o sistema de sangria deve ser modificado pelo aumento no intervalo entre sangrias e pela redução ou supressão de estimulação (Bernardes et al., 1995).

Segundo Conduru Neto (1986), a utilização de substâncias estimulantes que permitem o aumento do escoamento do látex após a sangria tem compensado o menor número de cortes adotados nos sistemas de baixa freqüência, atingindo os dois fatores básicos de produção: fisiológico e econômico. Atualmente, a estimulação é uma prática que vem sendo utilizada com freqüência nos seringais.

Nos últimos anos, vários trabalhos de pesquisa foram desenvolvidos sobre o desempenho de clones de seringueira submetidos a diferentes sistemas de sangria, destacando-se entre esses os de Gonçalves et al. (2000), Pereira et al. (2001) e Ferreira et al. (2002).

Este trabalho teve como objetivo identificar o melhor sistema de explotação do látex, para cada um dos três clones de seringueira na região oeste do Estado de São
Paulo, em termos de rentabilidade e de aspectos fisiológicos.

\section{Material e Métodos}

O experimento foi instalado na Fazenda Santa Gilda, do grupo Rodobéns Agrícola e Pecuária Ltda., no Município de Guararapes, região oeste do Estado de São Paulo, no

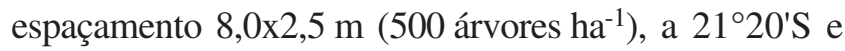
$50^{\circ} 50^{\prime} \mathrm{W}$ e altitude $560 \mathrm{~m}$, em Argissolo Vermelho eutrófico, arênico, profundo e bem drenado (Prado, 2003). Todos os tratos culturais ministrados ao experimento seguiram as recomendações técnicas para a cultura no Estado de São Paulo (Gonçalves et al., 2001).

Foi adotado o delineamento em blocos ao acaso, com parcelas subdivididas no tempo, com quatro repetições, de acordo com Steel \& Torrie (1980). A análise de variância foi realizada com o auxílio do SISVAR (Ferreira, 2003).

Os tratamentos principais foram os clones PR 255, RRIM 600 e GT 1, cada um alocado em parcelas de 0,25 ha em cada repetição. Os tratamentos secundários foram os sistemas de sangria: $1 / 2 \mathrm{~S} d / 26 \mathrm{~d} / 711 \mathrm{~m} / \mathrm{y}$ (testemunha) - sangria em meia espiral $(1 / 2 \mathrm{~S})$, realizada em intervalos de dois dias (d/2), com descanso aos domingos ( $6 \mathrm{~d} / 7)$, sangrando 11 meses por ano $(11 \mathrm{~m} / \mathrm{y}) ; 1 / 2 \mathrm{~S} \mathrm{~d} / 36 \mathrm{~d} / 7.11 \mathrm{~m} / \mathrm{y}$.ET 2,5\% Pa La $8 / \mathrm{y}-$ sangria em meia espiral $(1 / 2 \mathrm{~S})$, realizada em intervalos de três dias (d/3), com descanso aos domingos (6d/7), sangrando 11 meses por ano $(11 \mathrm{~m} / \mathrm{y})$, estimulado com ethephon a $2,5 \%$ (ET 2,5\%) aplicado no painel $(\mathrm{Pa})$ sobre a canaleta com cernambi (La), oito vezes por ano (8/y); $1 / 2 \mathrm{~S} \mathrm{~d} / 36 \mathrm{~d} / 7.11 \mathrm{~m} / \mathrm{y}$.ET 5\% Pa La 8/y - sangria em meia espiral $(1 / 2 \mathrm{~S})$, realizada em intervalos de três dias (d/3), com descanso aos domingos (6d/7), sangrando 11 meses por ano $(11 \mathrm{~m} / \mathrm{y})$, estimulado com ethephon a 5\% (ET 5\%) aplicado no painel (Pa) sobre a canaleta com cernambi (La), oito vezes por ano $(8 / \mathrm{y}) ; 1 / 2 \mathrm{~S} d / 46 \mathrm{~d} / 7.11 \mathrm{~m} / \mathrm{y}$.ET $2,5 \%$ $\mathrm{Pa} \mathrm{La} 8 / \mathrm{y}$ - sangria em meia espiral $(1 / 2 \mathrm{~S})$, realizada em intervalos de quatro dias (d/4), com descanso aos domingos (6d/7), sangrando 11 meses por ano $(11 \mathrm{~m} / \mathrm{y})$, estimulado com ethephon a $2,5 \%$ (ET 2,5\%) aplicado no painel $(\mathrm{Pa})$ sobre a canaleta com cernambi (La), oito vezes por ano (8/y); $1 / 2 \mathrm{~S} \mathrm{~d} / 46 \mathrm{~d} / 7.11 \mathrm{~m} / \mathrm{y}$.ET 5,0\% Pa La 8/y - sangria em meia espiral $(1 / 2 \mathrm{~S})$, realizada em intervalos de quatro dias $(\mathrm{d} / 4) \mathrm{com}$ 
descanso aos domingos (6d/7), sangrando 11 meses por ano $(11 \mathrm{~m} / \mathrm{y})$, com estimulação feita com ethephon a $5 \%$ (ET 5\%), aplicado no painel $(\mathrm{Pa})$ sobre a canaleta com cernambi $(\mathrm{La})$, oito vezes por ano $(8 / \mathrm{y}) ; 1 / 2 \mathrm{~S} \mathrm{~d} / 56 \mathrm{~d} / 7.11 \mathrm{~m} / \mathrm{y}$.ET $2,5 \%$ Pa La 8/y - sangria em meia espiral $(1 / 2 \mathrm{~S})$, realizada em intervalos de cinco dias (d/5), com descanso aos domingos (6d/7), sangrando 11 meses por ano $(11 \mathrm{~m} / \mathrm{y})$, com estimulação feita com 2,5\% de ethephon (ET 2,5\%), aplicado no painel (Pa) sobre a canaleta com cernambi (La), oito vezes por ano (8/y); $12 / 2 \mathrm{~S} \mathrm{~d} / 5$ 6d/7.11m/y.ET 5\% Pa La 8/y - sangria em meia espiral $(1 / 2 \mathrm{~S})$, realizada em intervalos de cinco dias $(\mathrm{d} / 5)$, com descanso aos domingos (6d/7), sangrando 11 meses por ano $(11 \mathrm{~m} / \mathrm{y})$, com estimulação feita com $5 \%$ de ethephon (ET 5\%), aplicado no painel (Pa) sobre a canaleta com cernambi (La), oito vezes por ano $(8 / \mathrm{y})$; $1 / 2 \mathrm{~S} \mathrm{~d} / 7$ 6d/7. 11m/y.ET 2,5\% Pa La 8/y - sangria em meia espiral $(1 / 2 \mathrm{~S})$, realizada em intervalos de sete dias (d/7), com descanso aos domingos (6d/7), sangrando 11 meses por ano ( $11 \mathrm{~m} / \mathrm{y})$, com estimulação feita com 2,5\% de ethephon (ET 2,5\%), aplicado no painel (Pa) sobre a canaleta com cernambi (La), oito vezes por ano (8/y); $1 / 2 \mathrm{~S} \mathrm{~d} / 76 \mathrm{~d} / 7.11 \mathrm{~m} / \mathrm{y}$.ET $5 \%$ Pa La 8/y - sangria em meia espiral $(1 / 2 \mathrm{~S})$, realizada em intervalos de sete dias $(\mathrm{d} / 7)$, com descanso aos domingos (6d/7), sangrando 11 meses por ano $(11 \mathrm{~m} / \mathrm{y})$, com estimulação feita com $5 \%$ de ethephon (ET 5\%), aplicado no painel (Pa) sobre a canaleta com cernambi (La), oito vezes por ano (8/y).

Foi avaliada mensalmente a produtividade de borracha no período adulto das árvores. O registro do rendimento foi efetuado pelo látex coagulado naturalmente nas tigelas. Houve adição de ácido acético a 5\% nos dias com ocorrência de chuva após a sangria. A massa total mensal de cada subparcela foi dividida pelo número total de coágulos, e os resultados foram expressos em gramas de borracha seca por corte por árvore. Com esses dados, estimou-se a produção por hectare ao ano por clone, em cada sistema de sangria. Na estimativa dos dados de produção de borracha, extrapolados para hectare por ano, adotaram-se, nos cálculos, estandes de 240, 340, 380 e 400 árvores em sangria por hectare no primeiro, segundo, terceiro, quarto e quinto ano, respectivamente (Embrapa, 1987). Nesta estimativa foram consideradas 140 sangrias por ano no sistema $1 / 2 \mathrm{~S} d / 2,104$ sangrias por ano no sistema $1 / 2 \mathrm{~S} \mathrm{~d} / 3 ; 78$ sangrias por ano no sistema $1 / 2 \mathrm{~S} \mathrm{~d} / 4 ; 62$ sangrias por ano no sistema $1 / 2 \mathrm{~S} d / 5$ e 52 sangrias por ano no sistema $1 \frac{1}{2} \mathrm{~S} d / 7$.
Ao final de cinco anos de avaliação, realizou-se o levantamento da incidência de árvores secas. Os painéis secos foram avaliados em cada sistema de sangria para cada clone e a porcentagem de painéis secos foi calculada de acordo com o número de plantas de cada sistema de sangria.

Nas parcelas, foram realizadas análises anuais com médias do perímetro do caule para avaliação do vigor das árvores. As mensurações foram conduzidas a 1,20 m do calo de enxertia, durante cinco anos de avaliação.

$\mathrm{Na}$ análise econômica foram estudados os seguintes parâmetros: receita bruta, salários, insumos, materiais agrícolas e, desta forma, foram determinados o custo operacional efetivo na produção, o lucro líquido e a rentabilidade de cada clone por sistema de explotação. Foram anotados os gastos anuais com insumos (ethephon e ácido acético), materiais agrícolas (tigelas, bicas, arame, faca de sangria e esmeril) para eventuais reposições e salários além dos encargos sociais, considerando que um seringueiro sangra 800 árvores por dia. O lucro líquido correspondeu à diferença entre a receita bruta e o custo total. A rentabilidade dos sistemas de sangria de cada clone foi calculada em relação à testemunha e expressa em porcentagem.

\section{Resultados e Discussão}

Na Tabela 1, é apresentado o resultado da análise de variância conjunta das médias de produtividade de borracha seca. Os coeficientes de variação estão dentro dos limites observados para a cultura da seringueira. Houve diferença significativa nos sistemas de sangria. As interações clones $\mathrm{x}$ anos, sistemas de sangria $\mathrm{x}$ anos e clones $\mathrm{x}$ sistemas de sangria $\mathrm{x}$ anos também foram significativas, porém, por serem de interesse secundário neste trabalho, não foram desdobradas.

Os resultados da análise anual das médias de produtividade de borracha encontram-se na Tabela 2. A precisão experimental foi boa uma vez que os coeficientes de variação são baixos. Os clones não apresentaram diferença significativa entre si em relação à produtividade. Houve interação significativa, em todos os anos, para clones x sistemas de sangria e, como esta 
interação é o interesse principal do estudo, foi realizada a análise do desdobramento (Tabela 3). Os sistemas de sangria, dentro de todos os clones e em todos os anos, apresentaram diferença significativa, pelo teste F.

As médias de produtividade de borracha estão presentes na Tabela 4. Observou-se superioridade dos sistemas $1 \frac{1}{2} \mathrm{~S} \mathrm{~d} / 3$.ET $2,5 \% 8 / \mathrm{y}$ e $1 / 2 \mathrm{~S}$ d/7.ET $2,5 \% 8 / \mathrm{y}$ para o clone GT 1 e do sistema $1 / 2 \mathrm{~S}$ d/3.ET 2,5\% 8/y para os clones PR 255 e RRIM 600 quando foi verificada a média de produtividade nos cinco anos de avaliação. Os sistemas de sangria $1 / 2 \mathrm{~S} d / 5$.ET 2,5\% 8/y e $1 / 2 \mathrm{~S} \mathrm{~d} / 5$.ET 5\% 8/y proporcionaram as menores médias de produtividade nos clones estudados, também considerando a produtividade média em cinco anos.

Tabela 1. Quadrados médios da análise de variância conjunta dos dados de produtividade de borracha seca $\left(\mathrm{kg} \mathrm{ha}^{-1}\right.$ por ano), de cinco anos de avaliação de três clones de seringueira, sob nove sistemas de sangria.

\begin{tabular}{lrc}
\hline Fonte de variação & GL & Quadrado médio \\
\hline Blocos & 3 & $520.834,39^{\text {ns }}$ \\
Clones (A) & 2 & $1.932 .733,63^{\text {ns }}$ \\
Resíduo (a) & 6 & $652.357,23$ \\
Sistemas de sangria (B) & 8 & $1.513 .014,06^{* *}$ \\
A x B & 16 & $163.447,62^{\text {ns }}$ \\
Resíduo (b) & 72 & $145.230,83$ \\
Anos (C) & 4 & $13.876 .461,72^{* *}$ \\
Ax C & 8 & $171.490,36^{* *}$ \\
B x C & 32 & $181.660,20^{* *}$ \\
Ax B x C & 64 & $23.979,27^{* *}$ \\
Resíduo (c) & 324 & $30.900,00$ \\
\hline Total & 539 & \\
\hline Média geral & $1.343,12$ & \\
CV (\%) (A) & 20,05 & \\
CV (\%) (B) & 28,37 & \\
CV (\%) (C) & 0,18 & \\
\hline
\end{tabular}

nsNão-significativo. **Significativo a $1 \%$ de probabilidade.
Na Tabela 5, é mostrado o perímetro médio anual do caule e os aumentos anuais neste perímetro, relativo aos cinco anos de avaliação referentes às médias de todos os subtratamentos para cada clone. De acordo com Gonçalves et al. (2006), a produtividade de borracha não necessariamente está correlacionada com $\mathrm{o}$ aumento do perímetro do caule. O clone com maior perímetro na abertura do painel de sangria foi o GT 1, seguido do RRIM 600 e PR 255. Esta característica do clone GT 1 é desejável, por caracterizar precocidade já que, de acordo com Gonçalves et al. (2001), as árvores são colocadas em sangria quando, além de outros critérios econômicos, apresentam pelo menos $45 \mathrm{~cm}$ de perímetro. Segundo esse mesmo autor, o vigor do clone GT 1, na Costa do Marfim, é baixo após sua entrada em sangria, mas em compensação é um clone muito homogêneo.

O clone com maior aumento no perímetro do caule, ao final de cinco anos de sangria, foi o clone PR 255, com aumento de $8,20 \mathrm{~cm}$. De acordo com Gonçalves et al. (1990), essa característica é importante dentro do programa de melhoramento genético da seringueira, pois as árvores continuam a crescer após a sangria, o que diminui a probabilidade de quebra por ventos.

Dados sobre o secamento do painel (Tabela 6) mostram que a incidência desse distúrbio fisiológico é diferente nos três clones estudados, o que confirma a teoria de que a sensibilidade à seca de painel é uma característica clonal, que pode ser influenciada, também, por vários fatores extrínsecos e intrínsecos à planta (Gonçalves et al., 2000; Usha Nair et al., 2004). Porém, as causas primárias do distúrbio ainda não foram esclarecidas, apesar de toda pesquisa desenvolvida sobre o assunto. Houve incidência

Tabela 2. Quadrados médios da análise de variância referente às médias de produtividade de borracha seca $\left(\mathrm{kg}\right.$ ha-1 $\left.\mathrm{por}^{-} \mathrm{ano}\right)$ de cinco anos, de três clones de seringueira, sob nove sistemas de sangria.

\begin{tabular}{|c|c|c|c|c|c|c|c|}
\hline \multirow[t]{2}{*}{ Fonte de variação } & \multirow[t]{2}{*}{ GL } & \multicolumn{6}{|c|}{ Sangrias anuais } \\
\hline & & $1^{\circ}$ ano & $2^{\underline{0}}$ ano & $3^{\circ}$ ano & $4^{\circ}$ ano & $5^{-0}$ ano & Média \\
\hline Blocos & 3 & $28.266,53^{\text {ns }}$ & $115.724,53^{\mathrm{ns}}$ & $137.450,41^{\mathrm{ns}}$ & $139.506,53^{\mathrm{ns}}$ & $130.868,33^{\mathrm{ns}}$ & $104.184,87^{\mathrm{ns}}$ \\
\hline Clones (A) & 2 & $62.389,49^{\text {ns }}$ & $577.120,04^{\mathrm{ns}}$ & $999.986,55^{\text {ns }}$ & $668.876,00^{\mathrm{ns}}$ & $310.322,99^{\mathrm{ns}}$ & $386.471,75^{\mathrm{ns}}$ \\
\hline Resíduo (a) & 6 & $36.300,70$ & $152.882,71$ & $177.572,08$ & $165.576,60$ & $157.612,70$ & $130.470,44$ \\
\hline Sistemas de sangria (B) & 8 & $88.778,80 * *$ & $287.057,01^{* *}$ & $405.901,69 * *$ & $662.870,83^{* *}$ & $795.046,52 * *$ & $302.591,85^{* *}$ \\
\hline $\mathrm{A} \times \mathrm{B}$ & 16 & $3.771,26^{* *}$ & $31.918,45^{* *}$ & $39.248,99 * *$ & $105.470,99 * *$ & $78.955,02 * *$ & $32.691,80 * *$ \\
\hline Resíduo (b) & 72 & 59,93 & 218,18 & 301,32 & 543,59 & 592,91 & 229,07 \\
\hline Total & 107 & & & & & & \\
\hline Média & & 702,52 & $1.442,26$ & $1.557,27$ & $1.523,92$ & $1.485,63$ & $1.343,12$ \\
\hline $\mathrm{CV}(\%)(\mathrm{A})$ & & 8,99 & 9,04 & 9,02 & 8,90 & 8,91 & 8,96 \\
\hline $\mathrm{CV}(\%)(\mathrm{B})$ & & 1,10 & 1,02 & 1,11 & 1,53 & 1,64 & 1,13 \\
\hline
\end{tabular}

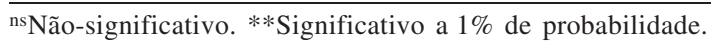


Tabela 3. Quadrados médios da análise de variância do desdobramento de sistema de sangria dentro de cada clone, de cinco anos de avaliação.

\begin{tabular}{|c|c|c|c|c|c|c|c|}
\hline \multirow[t]{2}{*}{ Fonte de variação } & \multirow[t]{2}{*}{ GL } & \multicolumn{6}{|c|}{ Quadrados médios } \\
\hline & & $1^{0}$ ano & $2^{-0}$ ano & $3^{-}$ano & $4^{\underline{0}}$ ano & $5^{-0}$ ano & Média \\
\hline Sistema de sangria/GT1 & 8 & $29.515,23 * *$ & $104.363,83^{* *}$ & $98.971,48^{* *}$ & $147.631,04^{* *}$ & $254.971,44^{* *}$ & $70.632,03 * *$ \\
\hline Sistema de sangria/PR255 & 8 & $37.514,93 * *$ & $63.643,21^{* *}$ & $194.884,06^{* *}$ & $498.096,18^{* *}$ & $373.902,60^{* *}$ & $156.583,16^{* *}$ \\
\hline Sistema de sangria/RRIM600 & 8 & $29.291,16^{* *}$ & $182.886,86^{* *}$ & $190.544,13^{* *}$ & $228.085,60^{* *}$ & $324.082,52^{* *}$ & $140.760,24^{* *}$ \\
\hline Resíduo & 72 & 59,93 & 218,18 & 301,32 & 543,60 & 592,91 & 229,07 \\
\hline
\end{tabular}

**Significativo a $1 \%$ de probabilidade,

Tabela 4. Produção anual de borracha seca $\left(\mathrm{kg} \mathrm{ha}^{-1}\right.$ por ano) referente a cinco anos de sangria de três clones de seringueira, sob diferentes sistemas de sangria(1). Sistemas de sangria

\begin{tabular}{|c|c|c|c|c|c|c|c|c|c|c|c|c|c|c|c|c|c|c|}
\hline \multirow[t]{2}{*}{ Sistemas de sangria } & \multicolumn{6}{|c|}{ GT 1} & \multicolumn{6}{|c|}{ PR 255} & \multicolumn{6}{|c|}{ RRIM 600} \\
\hline & $1^{\circ}$ ano & $2^{\circ}$ ano & $3^{\circ}$ ano & $4^{\circ}$ ano & $5^{\circ}$ ano & Média & $1^{\circ}$ ano & $2^{\circ}$ ano & $3^{\circ}$ ano & $4^{\circ}$ ano & $5^{\circ}$ ano & Média & $1^{\circ}$ ano & $2^{\circ}$ ano & $3^{\circ}$ ano & $4^{\circ}$ ano & $5^{\circ}$ ano & Média \\
\hline \multirow[t]{2}{*}{ 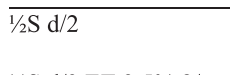 } & $691 \mathrm{~d}$ & $1.142 \mathrm{e}$ & $1.332 \mathrm{e}$ & $1.598 \mathrm{~b}$ & $1.802 \mathrm{a}$ & $1.313 \mathrm{~b}$ & $821 \mathrm{c}$ & $1.486 \mathrm{e}$ & $1.560 \mathrm{e}$ & $1.957 \mathrm{~b}$ & $1.949 \mathrm{~b}$ & $1.562 \mathrm{~b}$ & $757 \mathrm{~b}$ & $1.343 \mathrm{e}$ & $1.598 \mathrm{~d}$ & $1.845 \mathrm{~b}$ & $1.908 \mathrm{~b}$ & $1.490 \mathrm{c}$ \\
\hline & $(20,56)$ & $(23,99)$ & $(25,04)$ & $(28,54)$ & $(32,18)$ & $(26,06)$ & $(24,42)$ & $(31,21)$ & $(30,07)$ & $(34,95)$ & $(34,81)$ & $(31,09)$ & $(22,53)$ & $(28,22)$ & $(22,53)$ & $(28,22)$ & $(30,03)$ & $(26,30)$ \\
\hline \multirow[t]{2}{*}{$1 / 2 \mathrm{~S} \mathrm{~d} / 3$.ET 2,5\% 8/y } & $770 \mathrm{~b}$ & $1.544 a$ & $1.573 \mathrm{a}$ & $1.544 \mathrm{c}$ & $1.545 \mathrm{c}$ & $1.395 \mathrm{a}$ & $913 a$ & $1.769 a$ & & $2.176 \mathrm{a}$ & $2.090 \mathrm{a}$ & $1.806 \mathrm{a}$ & $804 a$ & $1.724 b$ & $1.985 a$ & $2.111 \mathrm{a}$ & $2.057 a$ & $1.736 \mathrm{a}$ \\
\hline & $(30,86)$ & $(43,66)$ & $(39,80)$ & $(37,12)$ & $(37,13)$ & $(37,71)$ & $(36,57)$ & $(50,02)$ & $(52,69)$ & $(52,32)$ & $(50,25)$ & $(48,37)$ & $(32,23)$ & $(48,75)$ & $(32,23)$ & $(48,75)$ & $(50,22)$ & $(42,44)$ \\
\hline \multirow[t]{2}{*}{ 1/2S d/3.ET 5,0\% 8/y } & $842 a$ & $1.566 \mathrm{a}$ & $1.466 \mathrm{c}$ & $1.330 \mathrm{e}$ & $1.231 \mathrm{f}$ & $1.287 \mathrm{bc}$ & $869 b$ & $1.724 \mathrm{~b}$ & $1.973 b$ & $1.824 \mathrm{c}$ & $1.587 \mathrm{c}$ & $1.596 \mathrm{~b}$ & $810 \mathrm{a}$ & $1.771 \mathrm{a}$ & $1.906 \mathrm{~b}$ & $1.815 b$ & $1.609 \mathrm{c}$ & $1.582 \mathrm{~b}$ \\
\hline & $(33,73)$ & $(44,30)$ & $(37,08)$ & $(31,99)$ & $(29,60)$ & $(35,34)$ & $(34,82)$ & $(48,77)$ & $(49,92)$ & $(43,84)$ & $(38,15)$ & $(43,10)$ & $(32,47)$ & $(50,09)$ & $(32,47)$ & $(50,09)$ & $(48,22)$ & $(42,67)$ \\
\hline \multirow[t]{2}{*}{ 1/2S d/4.ET 2,5\% 8/y } & $600 \mathrm{fg}$ & $1.234 \mathrm{~d}$ & $1.306 \mathrm{e}$ & $1.310 \mathrm{e}$ & $1.317 \mathrm{e}$ & $1.154 \mathrm{~d}$ & $679 \mathrm{ef}$ & $1.568 \mathrm{~d}$ & $1.755 \mathrm{c}$ & $1.631 \mathrm{~d}$ & $1.533 \mathrm{c}$ & $1.433 \mathrm{c}$ & $628 \mathrm{~d}$ & $1.397 \mathrm{~d}$ & $1.684 \mathrm{c}$ & $1.614 \mathrm{c}$ & $1.594 \mathrm{c}$ & $1.383 \mathrm{~d}$ \\
\hline & $(32,04)$ & $(46,53)$ & $(44,08)$ & $(41,98)$ & $(42,22)$ & $(41,37)$ & $(36,25)$ & $(59,11)$ & $(59,19)$ & $(52,26)$ & $(49,14)$ & $(51,19)$ & $(33,53)$ & $(52,67)$ & $(33,53)$ & $(52,67)$ & $(56,83)$ & $(45,85)$ \\
\hline \multirow[t]{2}{*}{$1 / 2 \mathrm{~S} \mathrm{~d} / 4$.ET $5,0 \% 8 / \mathrm{y}$} & $667 \mathrm{e}$ & $1.308 \mathrm{c}$ & $1.375 \mathrm{~d}$ & $1.236 \mathrm{f}$ & $1.203 \mathrm{fg}$ & $1.158 \mathrm{~d}$ & $676 \mathrm{ef}$ & $1.594 \mathrm{~d}$ & $1.663 \mathrm{~d}$ & $1.240 \mathrm{f}$ & $1.221 \mathrm{f}$ & $1.279 \mathrm{e}$ & $654 \mathrm{c}$ & $1.548 \mathrm{c}$ & $1.659 \mathrm{c}$ & $1.479 \mathrm{de}$ & $1.348 \mathrm{e}$ & $1.337 \mathrm{e}$ \\
\hline & $(35,61)$ & $(49,33)$ & $(46,38)$ & $(39,62)$ & $(38,57)$ & $(41,90)$ & $(36,13)$ & $(60,11)$ & $(56,10)$ & $(39,74)$ & $(39,13)$ & $(46$, & $(34,95)$ & $(58,36)$ & $(34,95)$ & & $(55,96)$ & $(48,52)$ \\
\hline \multirow[t]{2}{*}{ 1/2S d/5.ET 2,5\% 8/y } & $584 \mathrm{~g}$ & $1.096 \mathrm{f}$ & $1.106 \mathrm{~g}$ & $1.169 \mathrm{~g}$ & $1.172 \mathrm{~g}$ & $1.025 \mathrm{e}$ & $673 \mathrm{ef}$ & $1.396 \mathrm{f}$ & $1.415 \mathrm{~g}$ & $1.370 \mathrm{e}$ & $1.441 d$ & $1.259 \mathrm{ef}$ & $597 \mathrm{e}$ & $1.196 \mathrm{~g}$ & $1.389 \mathrm{f}$ & $1.531 \mathrm{~d}$ & $1.425 \mathrm{~d}$ & $1.228 \mathrm{f}$ \\
\hline & $(39,25)$ & $(51,99)$ & $(46,92)$ & $(47,13)$ & $(47,24)$ & $(46,51)$ & $(45,25)$ & $(66,20)$ & $(60,05)$ & $(55,24)$ & $(58,10)$ & & $(40,11)$ & & $(40,11)$ & $(56,75)$ & & $(50,54)$ \\
\hline \multirow[t]{2}{*}{$1 / 2 \mathrm{Sd} / 5$.ET 5,0\% 8/y } & $671 \mathrm{e}$ & $1.281 \mathrm{c}$ & $1.173 \mathrm{f}$ & $1.108 \mathrm{~h}$ & $1.053 \mathrm{~h}$ & $1.057 \mathrm{e}$ & $691 \mathrm{e}$ & $1.583 \mathrm{~d}$ & $1.507 \mathrm{f}$ & $1.203 \mathrm{f}$ & $1.203 \mathrm{f}$ & $1.237 \mathrm{f}$ & $628 \mathrm{~d}$ & $1.394 \mathrm{~d}$ & $1.352 \mathrm{f}$ & $1.366 \mathrm{f}$ & $1.164 \mathrm{f}$ & $1.181 \mathrm{~g}$ \\
\hline & $(45,10)$ & $(60,77)$ & $(49,79)$ & $(44,66)$ & $(42,47)$ & $(48,5$ & $(46,42)$ & $(75,08)$ & $(63,94)$ & $(48,52)$ & $(48,51)$ & $(56$, & $(42,23)$ & $(66,11)$ & $(42,23)$ & & $(57,38)$ & $(54,81)$ \\
\hline \multirow[t]{2}{*}{ 1/2S d/7.ET 2,5\% 8/y } & $602 \mathrm{f}$ & $1.367 \mathrm{~b}$ & $1.525 \mathrm{~b}$ & $1.651 \mathrm{a}$ & $1.692 \mathrm{~b}$ & $1.367 \mathrm{a}$ & $667 \mathrm{~g}$ & $1.427 \mathrm{f}$ & $1.507 \mathrm{f}$ & $1.253 \mathrm{f}$ & $1.338 \mathrm{e}$ & $1.238 \mathrm{f}$ & $601 \mathrm{e}$ & $1.211 \mathrm{fg}$ & $1.471 \mathrm{e}$ & $1.508 \mathrm{de}$ & $1.453 \mathrm{~d}$ & $1.249 \mathrm{f}$ \\
\hline & $(48,20)$ & $(77,29)$ & $(77,20)$ & $(79,39)$ & $(81,32)$ & $(72,68)$ & $(53,45)$ & $(80,73)$ & $(76,28)$ & $(60,22)$ & $(64,32)$ & $(67$, & $(48,18)$ & $(68,51)$ & $(48,18)$ & $(68,51)$ & $(74,44)$ & $(61,56)$ \\
\hline \multirow[t]{2}{*}{$1 / 2 \mathrm{~S}$ d/7.ET 5,0\% 8/y } & $731 \mathrm{c}$ & $1.391 \mathrm{~b}$ & $1.446 \mathrm{c}$ & $1.407 \mathrm{~d}$ & $1.392 \mathrm{~d}$ & $1.278 \mathrm{c}$ & $803 \mathrm{~d}$ & $1.645 \mathrm{c}$ & $1.675 \mathrm{~d}$ & $1.406 \mathrm{e}$ & $1.443 \mathrm{~d}$ & $1.394 \mathrm{~d}$ & $648 \mathrm{c}$ & $1.238 \mathrm{f}$ & $1.506 \mathrm{e}$ & $1.465 \mathrm{e}$ & $1.343 \mathrm{e}$ & $1.240 \mathrm{f}$ \\
\hline & $(58,61)$ & $(78,67)$ & $(74,20)$ & $(67,63)$ & $(66,94)$ & $(69,21)$ & $(64,31)$ & $(93,07)$ & $(84,75)$ & $(67,60)$ & $(69,36)$ & $(75,82)$ & $(51,89)$ & $(70,01)$ & $(51,89)$ & $(70,01)$ & $(76,21)$ & $(64,00)$ \\
\hline
\end{tabular}

${ }^{(1)}$ Médias seguidas de mesma letra, nas colunas, não diferem entre si pelo teste de Tukey, a 1\% de probabilidade; valores entre parênteses referem-se aos dados em gramas por árvore por sangria. 
de mais de $5 \%$ de seca do painel em alguns sistemas de sangria, como no $1 / 2 \mathrm{~S} d / 3$.ET $5 \%$ 8/y que mostrou incidência de 12,7, 5,8 e 7,7\% sobre o total do estande, nos clones GT 1, PR 255 e RRIM 600, respectivamente. Estes dados evidenciam a importância da função técnica no gerenciamento do seringal para uma exploração racional, pois de acordo com a Secretaria de Agricultura e Abastecimento do Estado de São Paulo (São Paulo, 1999), atingidos 5\% das plantas, é necessária uma análise e revisão do método de exploração empregado. O clone GT 1 foi o que apresentou a maior suscetibilidade à seca do painel. Este resultado discorda dos relatados por Pinheiro et al. (2002) quanto aos dados obtidos sobre a suscetibilidade à seca do painel de clones do seringal comercial da Codeara, em Mato Grosso, que mostraram que o clone GT 1 apresentou $0,1 \%$ de incidência do distúrbio sobre as árvores em corte, o que provavelmente pode ser devido à interação genótipo-ambiente. Houve menor incidência de seca do painel no sistema de

Tabela 5. Perímetro do caule de cinco anos de sangria referente a três clones de seringueira ${ }^{(1)}$.

\begin{tabular}{lccccc}
\hline Clones & \multicolumn{5}{c}{ Perímetro do caule $(\mathrm{cm})$} \\
\cline { 2 - 6 } & $1^{\underline{0}}$ ano $^{(2)}$ & $2^{\underline{0}}$ ano & $3^{\underline{0}}$ ano & $4^{\underline{0}}$ ano & $5^{\underline{0}}$ ano \\
\hline \multirow{2}{*}{ GT 1 } & 55,60 & 56,80 & 57,60 & 58,60 & 60,40 \\
\multirow{2}{*}{ PR 255 } & & $(1,20)$ & $(0,80)$ & $(1,00)$ & $(1,80)$ \\
& \multirow{2}{*}{ RRIM 600 } & 52,00 & 53,20 & 54,60 & 58,40 \\
& \multirow{2}{*}{50,80} & $(1,80)$ & $(1,20)$ & $(1,40)$ & $(3,80)$ \\
& & 53,80 & 55,20 & 56,40 & 58,80 \\
& $(3,00)$ & $(1,40)$ & $(1,20)$ & $(2,40)$ \\
\hline
\end{tabular}

(1) Valores referem-se à média de todos os sistemas de sangria para cada clone; valores entre parênteses referem-se ao aumento de perímetro do caule de um ano em relação ao anterior. ${ }^{(2)}$ Ano correspondente à abertura do painel de sangria. sangria $1 / 2 \mathrm{~S}$ d/7.ET $5 \% 8 / \mathrm{y}$ em todos os clones estudados, o que reforça a teoria de que sistemas de explotação com baixa frequiência de sangria reduzem a possibilidade de ocorrência desse distúrbio (Gonçalves et al., 2001).

Pela análise econômica dos diferentes sistemas de sangria dos três clones de seringueira, com base nos cinco anos de avaliação, percebe-se que a rentabilidade dos clones PR 255 e RRIM 600, no sistema $1 \frac{1}{2} \mathrm{~S}$ d/3. ET 2,5\% 8/y, foi superior em 43 e 47\%, respectivamente, em relação à testemunha (Tabela 7). Já a maior rentabilidade do clone GT 1 foi alcançada no sistema $1 \frac{1}{2} \mathrm{~S}$ d/7.ET 2,5\% 8/y, em que observouse superioridade de $61 \%$ em relação ao sistema $1 / 2 \mathrm{~S} \mathrm{~d} / 2$ (testemunha). De acordo com Pinheiro et al. (2002), na freqüência d/7 um seringueiro pode sangrar até 7.000 árvores, conferindo economia de até $32 \%$ sobre o item de despesa com mão-de-obra.

Esses sistemas de explotação, nos clones estudados, além de apresentarem maiores rendimentos financeiros, proporcionaram menor incidência de seca do painel, portanto sua utilização é viável, tanto sob o aspecto econômico quanto fisiológico. Alguns sistemas de sangria apresentaram rentabilidade menor que a testemunha como o $1 / 2 \mathrm{~S}$ d/5.ET 5\% 8/y nos clones PR 255 e RRIM 600, devido à baixa produtividade obtida nestes sistemas. Sugere-se, também, o uso, em plantios comerciais, do sistema $1 \frac{1}{2} \mathrm{~S}$ d/7.ET $5 \%$ 8/y no clone GT 1 , por ter apresentado bom resultado econômico e baixa incidência de seca do painel.

Tabela 6. Incidência de secamento do painel de três clones de seringueira no 5o ano de avaliação em diferentes sistemas de sangria.

\begin{tabular}{|c|c|c|c|c|c|c|c|c|c|}
\hline \multirow[t]{2}{*}{ Sistemas de sangria } & \multicolumn{3}{|c|}{ GT1 } & \multicolumn{3}{|c|}{ PR 255} & \multicolumn{3}{|c|}{ RRIM 600} \\
\hline & $\begin{array}{l}\text { No total de } \\
\text { plantas } \\
\text { sangradas }\end{array}$ & $\begin{array}{l}\text { Total de } \\
\text { plantas } \\
\text { secas }\end{array}$ & $\begin{array}{c}\text { Porcentagens } \\
\text { de seca do } \\
\text { painel }\end{array}$ & $\begin{array}{l}\text { № total de } \\
\text { plantas } \\
\text { sangradas }\end{array}$ & $\begin{array}{l}\text { Total de } \\
\text { plantas } \\
\text { secas }\end{array}$ & $\begin{array}{c}\text { Porcentagens } \\
\text { de seca do } \\
\text { painel }\end{array}$ & $\begin{array}{l}\text { No total de } \\
\text { plantas } \\
\text { sangradas }\end{array}$ & $\begin{array}{l}\text { Total de } \\
\text { plantas } \\
\text { secas }\end{array}$ & $\begin{array}{c}\text { Porcentagens } \\
\text { de seca do } \\
\text { painel }\end{array}$ \\
\hline $1 / 2 \mathrm{~S} \mathrm{~d} / 2$ (test.) & 90 & 7 & 7,2 & 97 & 3 & 3,0 & 94 & 4 & 4,1 \\
\hline $1 / 2 \mathrm{~S} \mathrm{~d} / 3$.ET $2,5 \% 8 / \mathrm{y}$ & 47 & 4 & 7,8 & 48 & 1 & 2,0 & 47 & 1 & 2,1 \\
\hline $1 / 2 \mathrm{~S} \mathrm{~d} / 3$. ET $5,0 \% 8 / \mathrm{y}$ & 48 & 7 & 12,7 & 49 & 3 & 5,8 & 48 & 4 & 7,7 \\
\hline $1 / 2 \mathrm{~S} \mathrm{~d} / 4 . \mathrm{ET} 2,5 \% 8 / \mathrm{y}$ & 44 & 7 & 13,7 & 46 & 2 & 4,2 & 46 & 1 & 2,1 \\
\hline $1 / 2 \mathrm{~S} \mathrm{~d} / 4$.ET $5,0 \% 8 / \mathrm{y}$ & 48 & 2 & 4,0 & 46 & 3 & 6,1 & 45 & 4 & 8,2 \\
\hline $1 / 2 \mathrm{~S} \mathrm{~d} / 5$.ET $2,5 \% 8 / \mathrm{y}$ & 49 & 2 & 3,9 & 49 & - & - & 46 & - & - \\
\hline $1 / 2 \mathrm{~S} \mathrm{~d} / 5$.ET $5,0 \% 8 / \mathrm{y}$ & 49 & 2 & 3,9 & 50 & 2 & 3,8 & 47 & 1 & 2,1 \\
\hline $1 / 2 \mathrm{~S} \mathrm{~d} / 7 . \mathrm{ET} 2,5 \% 8 / \mathrm{y}$ & 49 & 1 & 2,0 & 45 & 4 & 8,2 & 46 & - & - \\
\hline $1 / 2 \mathrm{~S} \mathrm{~d} / 7$.ET $5,0 \% 8 / \mathrm{y}$ & 48 & 1 & 2,0 & 47 & - & - & 45 & - & - \\
\hline
\end{tabular}


Tabela 7. Análise econômica de diferentes sistemas de sangria de três clones de seringueira, com base na média de produtividade de borracha seca de cinco anos de avaliação.

\begin{tabular}{|c|c|c|c|c|c|c|c|c|c|}
\hline Clones & $\begin{array}{l}\text { Sistemas } \\
\text { de sangria }\end{array}$ & $\begin{array}{l}\text { Produtividade } \\
\qquad\left(\mathrm{kg} \mathrm{ha}^{-1}\right)\end{array}$ & $\begin{array}{l}\text { Receita bruta } \\
(\mathrm{R} \$)^{(1)}\end{array}$ & $\begin{array}{l}\text { Mão-de-obra } \\
(\mathrm{RS})^{(2)}\end{array}$ & $\begin{array}{l}\text { Insumos } \\
(\mathrm{R} \$)^{(3)}\end{array}$ & $\begin{array}{l}\text { Materiais } \\
\text { agrícolas } \\
(\mathrm{R} \$)^{(4)}\end{array}$ & $\begin{array}{c}\text { Custos } \\
\text { operacionais } \\
\text { efetivos (R\$) }\end{array}$ & $\begin{array}{l}\text { Receita } \\
\text { liquida } \\
\text { (RS) }\end{array}$ & $\begin{array}{c}\text { Rentabilidade } \\
(\%)^{(5)}\end{array}$ \\
\hline \multirow[t]{9}{*}{ GT1 } & $1 / 2 \mathrm{~S} \mathrm{~d} / 2$ (test.) & $1.313,06$ & $4.595,71$ & $2.112,50$ & 10,78 & 35,00 & $2.158,28$ & $2.437,43$ & 100 \\
\hline & $1 / 2 \mathrm{~S} \mathrm{~d} / 3$.ET $2,5 \% 8 / y$ & $1.395,10$ & $4.882,86$ & $1.408,33$ & 126,37 & 35,00 & $1.569,70$ & $3.313,16$ & 136 \\
\hline & $1 / 2 \mathrm{~S} \mathrm{~d} / 3$.ET $5,0 \% 8 / y$ & $1.287,16$ & $4.505,05$ & $1.408,33$ & 241,95 & 35,00 & $1.685,28$ & $2.819,77$ & 116 \\
\hline & $1 / 2 \mathrm{~S} \mathrm{~d} / 4 . \mathrm{ET} 2,5 \% 8 / \mathrm{y}$ & $1.153,49$ & $4.037,22$ & $1.056,25$ & 126,37 & 35,00 & $1.217,62$ & $2.819,60$ & 116 \\
\hline & $1 / 2 \mathrm{~S} \mathrm{~d} / 4$.ET $5,0 \% 8 / \mathrm{y}$ & $1.157,87$ & $4.052,56$ & $1.056,25$ & 241,95 & 35,00 & $1.333,20$ & $2.719,36$ & 112 \\
\hline & $1 / 2 \mathrm{~S} \mathrm{~d} / 5 . \mathrm{ET} 2,5 \% 8 / \mathrm{y}$ & $1.025,16$ & $3.588,07$ & 845,00 & 126,37 & 35,00 & $1.006,37$ & $2.581,70$ & 106 \\
\hline & $1 / 2 \mathrm{~S} \mathrm{~d} / 5 . \mathrm{ET} 5,0 \% 8 / \mathrm{y}$ & $1.057,14$ & $3.699,98$ & 845,00 & 241,95 & 35,00 & $1.121,95$ & $2.578,03$ & 106 \\
\hline & $1 / 2 \mathrm{~S} \mathrm{~d} / 7 . \mathrm{ET} 2,5 \% 8 / \mathrm{y}$ & $1.367,37$ & $4.785,78$ & 704,17 & 126,37 & 35,00 & 865,53 & $3.920,25$ & 161 \\
\hline & $1 / 2 \mathrm{~S} \mathrm{~d} / 7$. ET $5,0 \% 8 / \mathrm{y}$ & $1.277,41$ & $4.470,94$ & 704,17 & 244,76 & 35,00 & 983,93 & $3.487,01$ & 143 \\
\hline \multirow[t]{9}{*}{ PR 255} & $1 / 2 \mathrm{~S} \mathrm{~d} / 2$ (test.) & $1.562,28$ & $5.467,99$ & $2.112,50$ & 11,03 & 35,00 & $2.158,53$ & $3.309,45$ & 100 \\
\hline & $1 / 2 \mathrm{~S} \mathrm{~d} / 3$. ET $2,5 \% 8 / y$ & $1.806,15$ & $6.321,54$ & $1.408,33$ & 129,31 & 35,00 & $1.572,64$ & $4.748,90$ & 143 \\
\hline & $1 / 2 \mathrm{~S} \mathrm{~d} / 3$.ET $5,0 \% 8 / \mathrm{y}$ & $1.595,45$ & $5.584,09$ & $1.408,33$ & 247,58 & 35,00 & $1.690,91$ & $3.893,18$ & 118 \\
\hline & $1 / 2 \mathrm{~S} \mathrm{~d} / 4 . \mathrm{ET} 2,5 \% 8 / \mathrm{y}$ & $1.432,93$ & $5.015,24$ & $1.056,25$ & 129,31 & 35,00 & $1.220,56$ & $3.794,69$ & 115 \\
\hline & $1 / 2 \mathrm{~S} \mathrm{~d} / 4$.ET $5,0 \% 8 / \mathrm{y}$ & $1.278,72$ & $4.475,53$ & $1.056,25$ & 247,58 & 35,00 & $1.338,83$ & $3.136,70$ & 95 \\
\hline & $1 / 2 \mathrm{~S} \mathrm{~d} / 5$. ET $2,5 \% 8 / y$ & $1.258,82$ & $4.405,86$ & 845,00 & 129,31 & 35,00 & $1.009,31$ & $3.396,55$ & 103 \\
\hline & $1 / 2 \mathrm{~S} \mathrm{~d} / 5$.ET $5,0 \% 8 / \mathrm{y}$ & $1.237,31$ & $4.330,59$ & 845,00 & 247,58 & 35,00 & $1.127,58$ & $3.203,02$ & 97 \\
\hline & $1 / 2 \mathrm{~S} \mathrm{~d} / 7$. ET $2,5 \% 8 / \mathrm{y}$ & $1.238,39$ & $4.334,36$ & 704,17 & 129,31 & 35,00 & 868,47 & $3.465,88$ & 105 \\
\hline & $1 / 2 \mathrm{~S} \mathrm{~d} / 7 . \mathrm{ET} 5,0 \% 8 / \mathrm{y}$ & $1.394,39$ & $4.880,37$ & 704,17 & 247,58 & 35,00 & 986,74 & $3.893,62$ & 118 \\
\hline \multirow[t]{9}{*}{ RRIM 600} & $1 / 2 \mathrm{~S} \mathrm{~d} / 2$ (test.) & $1.490,10$ & $5.215,36$ & $2.112,50$ & 10,78 & 35,00 & $2.158,28$ & $3.057,08$ & 100 \\
\hline & $1 / 2 \mathrm{~S} \mathrm{~d} / 3 . \mathrm{ET} 2,5 \% 8 / \mathrm{y}$ & $1.736,05$ & $6.076,19$ & $1.408,33$ & 126,37 & 35,00 & $1.569,70$ & $4.506,49$ & 147 \\
\hline & $1 / 2 \mathrm{~S} \mathrm{~d} / 3 . \operatorname{ET} 5,0 \% 8 / \mathrm{y}$ & $1.582,24$ & $5.537,83$ & $1.408,33$ & 241,95 & 35,00 & $1.685,28$ & $3.852,55$ & 126 \\
\hline & $1 / 2 \mathrm{~S} \mathrm{~d} / 4 . \mathrm{ET} 2,5 \% 8 / \mathrm{y}$ & $1.383,45$ & $4.842,09$ & $1.056,25$ & 126,37 & 35,00 & $1.217,62$ & $3.624,47$ & 119 \\
\hline & $1 / 2 \mathrm{~S} \mathrm{~d} / 4$.ET $5,0 \% 8 / \mathrm{y}$ & $1.337,36$ & $4.680,75$ & $1.056,25$ & 241,95 & 35,00 & $1.333,20$ & $3.347,55$ & 110 \\
\hline & $1 / 2 \mathrm{~S} \mathrm{~d} / 5$. ET $2,5 \% 8 / y$ & $1.227,53$ & $4.296,36$ & 845,00 & 126,37 & 35,00 & $1.006,37$ & $3.289,99$ & 108 \\
\hline & $1 / 2 \mathrm{~S} \mathrm{~d} / 5$.ET $5,0 \% 8 / \mathrm{y}$ & $1.180,72$ & $4.132,51$ & 845,00 & 241,95 & 35,00 & $1.121,95$ & $3.010,56$ & 98 \\
\hline & $1 / 2 \mathrm{~S} \mathrm{~d} / 7 . \mathrm{ET} 2,5 \% 8 / \mathrm{y}$ & $1.248,67$ & $4.370,34$ & 704,17 & 126,37 & 35,00 & 865,53 & $3.504,81$ & 115 \\
\hline & $1 / 2 \mathrm{~S} \mathrm{~d} / 7$. ET $5,0 \% 8 / \mathrm{y}$ & $1.239,91$ & $4.339,69$ & 704,17 & 244,76 & 35,00 & 983,93 & $3.355,76$ & 110 \\
\hline
\end{tabular}

${ }^{(1)} \mathrm{O}$ produto foi comercializado em forma de borracha seca em março de 2006 por $\mathrm{R} \$ 3,50$. ${ }^{(2)} \mathrm{O}$ salário base de R $\$ 650,00$, incluído $30 \%$ de encargos sociais por seringueiro ao mês, considerando que um seringueiro no sistema d/2 é responsável por 1.600 árvores; em d/3 por 2.400 árvores; em d/4 por 3.200 árvores; em d/5 por 4.000 árvores e em d/7 por 5.600 árvores. ${ }^{(3)}$ Verba destinada para a compra de ácido acético e ethrel de acordo com a necessidade de cada sistema de sangria. ${ }^{(4)}$ Verba destinada para a reposição de tigelas, bicas, arame, faca de sangria e esmeril, no caso de dano. ${ }^{(5)} \mathrm{A}$ porcentagem de rentabilidade é em relação à testemunha $(1 / 2 \mathrm{~S} d / 2)$, individual para cada clone.

\section{Conclusões}

1. Em relação à média de produtividade, há superioridade dos sistemas $1 / 2 \mathrm{~S} \mathrm{~d} / 3$.ET $2,5 \% 8 / \mathrm{y}$ e $1 / 2 \mathrm{~S}$ d/7. ET 2,5\% 8/y para o clone GT 1 e $1 / 2$ S d/3.ET 2,5\% 8/y para os clones PR 255 e RRIM 600.

2. O sistema $1 / 2 \mathrm{~S}$ d/3.ET $5 \% 8 / y$ necessita de uma revisão devido à elevada incidência de seca do painel.

3. Sistemas de explotação com baixa freqüência de sangria reduzem a possibilidade de ocorrência de seca do painel.

4. Os sistemas de explotação $1 \frac{1}{2} \mathrm{~S}$ d/3.ET 2,5\% 8/y, nos clones PR 255 e RRIM 600, e 1/2S d/7.ET 2,5\% 8/y, no clone GT 1, são superiores tanto do ponto de vista econômico quanto fisiológico.

\section{Agradecimentos}

Aos funcionários da Fazenda Santa Gilda, do grupo Rodobéns Agrícola e Pecuária Ltda., por propiciarem o desenvolvimento dos trabalhos.

\section{Referências}

BERNARDES, M.S.; CASTRO, P.R. de C. e; FURTADO, E.L.; SILVEIRA, A.P. da; COSTA, J.D.; MARTINS, A.N.; VIRGENS FILHO, A.C. Manual de sangria da seringueira. São José do Rio Preto: Bridgestone/Firestone do Brasil, 1995. 20p.

BERNARDES, M.S.; CASTRO, P.R. de C. e; MARTINS, A.N.; VIRGENS FILHO, A. de C. Fatores ligados à escolha do sistema de explotação. In: BERNARDES, M.S. (Ed.). Sangria da seringueira. Piracicaba: Esalq/USP, 2000. p.139-182.

CONDURU NETO, J.M.H. Sistema de explotação com freqüência reduzida de sangria e uso de estimulantes. In: ENCONTRO NACIONAL SOBRE EXPLOTAÇÃO E ORGANIZAÇÃO DE SERINGAIS DE CULTIVO, 1., 1986, Brasília. Anais. Brasília: Sudhevea, 1986. p.45-58.

EMBRAPA. Centro Nacional de Pesquisa de Seringueira e Dendê (Manaus, AM). Melhoramento genético da seringueira. Manaus: Embrapa-CNPSD, 1987. 23p. (Embrapa-CNPSD. Documentos, 10). FERREIRA, D.F. Sisvar. Versão 4.6 (Build 63). Lavras: DEX/Ufla, 2003. 1 CD-ROM.

FERREIRA, M.; MORENO, R.M.B.; GONÇALVES, P. de S.; MATTOSO, L.H.C. Evaluation of natural rubber from clones of 
Hevea brasiliensis. Rubber Chemistry and Technology, v.75, p.171-177, 2002.

GONÇALVES, P. de S.; BATAGLIA, O.C.; ORTOLANI, A.A.; FONSECA, F. da S. Manual de heveicultura para o Estado de São Paulo. Campinas: Instituto Agronômico, 2001. 78p.

GONÇALVES, P. de S.; CARDOSO, M.; ORTOLANI, A.A. Origem, variabilidade e domesticação da Hevea: uma revisão. Pesquisa Agropecuária Brasileira, v.25, p.135-156, 1990.

GONÇALVES, P. de S.; SILVA, M. de A.; GOUVÊA, L.R.L.; SCALOPPI JUNIOR, E.J. Genetic variability for girth growth and rubber yield characters in Hevea brasiliensis. Scientia Agricola, v.63, p.246-254, 2006.

GONÇALVES, P. de S.; SOUZA, S.R. de; BRIOSCHI, A.P.; VIRGENS FILHO, A. de C.; MAY, A.; CAPELA-ALARCON, R.S. Efeito da freqüência de sangria e estimulação no desempenho produtivo e econômico de clones de seringueira. Pesquisa Agropecuária Brasileira, v.35, p.1081-1091, 2000.

PEREIRA, A.V.; PEREIRA, E.B.C.; BENESI, J.F.C. Desempenho de clones de seringueira sob diferentes sistemas de sangria.
Planaltina: Embrapa Cerrados, 2001. 19p. (Embrapa Cerrados. Boletim de pesquisa e desenvolvimento, 16).

PINHEIRO, E.; CONCEIÇÃO, H.E.O.; PINHEIRO, F.S.V.; VIÉGAS, J.M.; ARANTES, M.A.L. A reabilitação da seringueira na Amazônia. In: CICLO DE PALESTRAS SOBRE A HEVEICULTURA PAULISTA, 3., 2002, São José do Rio Preto. Anais. São José do Rio Preto: SAA, Apabor, 2002. p.38-62.

PRADO, H. do. Solos do Brasil: gênese, morfologia, classificação, levantamento, manejo agrícola e geotécnico. 3.ed. Piracicaba: H. do Prado, 2003. 275p.

SÃO PAULO. Secretaria de Agricultura e Abastecimento. Comissão Técnica de Seringueira. A cultura da seringueira para o estado de São Paulo. Campinas: Cati, 1999. 90p.

STEEL, R.G.D.; TORRIE, J.H. Principles and procedures of statistics. New York: McGraw-Hill, 1980. 631p.

USHA NAIR, N.; RAMESH NAIR, B.; THOMAS, M.; GOPALAKRISHNAN, J. Latex diagnosis in relation to exploitation systems in clone RRII 105. Journal of Rubber Research, v.7, p.127-137, 2004.

Recebido em 15 de agosto de 2006 e aprovado em 23 de novembro de 2006 